Classification

Physics Abstracts

$02.40-61.40 \mathrm{D}-61.70$

\title{
Dual properties of conjugate disclination segment networks in amorphous materials
}

\author{
M. Kléman \\ Laboratoire de Physique des Solides (*), Bât. 510, 91405 Orsay, France
}

(Reçu le 22 décembre 1982, accepté le 23 février 1983)

\begin{abstract}
Résumé. - Nous étendons certains résultats récents concernant les propriétés structurales des matériaux amorphes déduites d'un modèle où les effets de frustration qui conduisent à un ordre local non cristallographique sont décrits par des espaces courbes [1]. On propose que la structure finale, obtenue à partir du " meilleur » choix d'application de l'espace courbe sur l'espace ordinaire, consiste en deux réseaux conjugués irréguliers formés de segments rectilignes. Ces deux réseaux sont des sources de distorsions de signes opposés, du type disinclinaison élastique. En outre, chaque réseau est de nature sphérique ou hyperbolique, en ce sens que l'on peut attacher à chacun d'eux des nombres de coordination différents pour les atomes. La taille typique $\xi$ d'un élément de réseau peut être estimée à partir de l'analyse d'effets compétitifs de contraintes élastiques internes et d'énergies de coeur, à basse température; à des températures plus élevées (au-dessus d'une température typique $T_{0}$ nettement plus faible que $T_{\mathrm{m}}$ ), les réseaux sont mobiles et l'on doit prendre en compte leur entropie, ce qui conduit à une variation de $\xi$ avec la température. On souligne les analogies et différences avec la loi de VogelFulcher usuelle, ainsi que l'existence d'un certain ordre orientationnel à longue distance.
\end{abstract}

\begin{abstract}
We extend some recent results concerning the structural properties of amorphous materials deduced from a model in which the frustration effects leading to non-crystallographic shortrange order are described by curved spaces [1]. It is proposed that the final structure obtained from the " best " mapping from curved space consists in two conjugate irregular networks of straight segments, which are sources of distortions of opposite signs and of the elastic disclination type. Also, each network carries either spherical or hyperbolic curvature, in the sense that they correspond to different coordination numbers for the atoms. The typical size $\xi$ of the network unit is estimated from a balance of (internal) elastic stresses and of core energies, at low temperature; at higher temperatures (above a typical $T_{0}$ temperature definitely smaller than $T_{\mathrm{m}}$ ) the networks are mobile and their entropy must be taken into account, which leads to a dependence of $\xi$ with temperature. Analogy and differences with a usual Vogel-Fulcher law are outlined, and the existence of some sort of long-range orientational order stressed.
\end{abstract}

In this paper we wish to deepen and extend some ideas put forward in a recent publication [1] concerning the structural properties of amorphous bodies. The model used very special geometrical methods which consist in describing a disordered system as a result of a decurving process of a

(*) Laboratoire associé au CNRS. 
space of negative curvature endowed with a regular lattice. We shall briefly recall the steps of this process, then show, as new results, that it leads, when it is « best " performed (from an energetical point of view), to an amorphous body possessing two irregular conjugated lattices of connected straight segments, each lattice being the source of elastic stresses of the disclination type and of opposite signs. This result points also at the duality between models using negative or positive curved spaces. The typical size of the lattice unit will be estimated at $0 \mathrm{~K}$, as well as the influence of configurational entropy in the supercooled state, i.e. above a typical temperature $T_{0}\left(<T_{\mathrm{g}}\right)$ which will appear in the theory.

\section{Frustration and curved spaces.}

Disorder in amorphous metals is believed to be due to the tendency of atoms to fill space as densely as possible [2]. Locally atoms would pack in regular tetrahedra, but such units cannot tile space in a uniform manner [3]. This frustration effect can be best described by introducing a spherical space, endowed with a regular lattice whose unit pattern consists in 5 tetrahedra meeting along a common edge; the small deficit angle which shows up when 5 regular tetrahedra meet this way in (flat) space vanishes in spherical space. And indeed five fold arrangements of tetrahedra have been observed in amorphous systems (in our usual flat space [4]) where they form the vast majority of polyhedra present in the body; these tetrahedra are of course slightly distorted with respect to regular tetrahedra. But it is desirable to consider that such a local arrangement of atoms, frustrated in the long range, belongs to the realm of spherical geometry. We say that the (real) medium displays spherical « curvature » locally.

Conversely [5], in amorphous systems tetracoordinated covalent atoms like to assemble in 7-member rings. Heptagons can tile a hyperbolic plane, with 3 of them at each vertex. The deficit angle $\omega=2 \pi-\frac{3 \times 5 \pi}{7}$ is now negative (slight recovering); it is reasonable to speak, in real space, of $2 \mathrm{~d}$ hyperbolic curvature. Note that the global description of frustration in this case would require two other curvatures, in $3 \mathrm{~d}$, while in the former case the curvature was the same in all sections of space (space of constant curvature).

The relationship between curvature and frustration was first proposed by Toulouse [6], but in the context of spin-glasses, not disordered atomic systems. The correspondences between these two uses of the concept certainly exist but have still to be worked out ; on the other hand, there is an evident similarity between our approach and Bilby's theory of representation of densities of disclinations by curved spaces [7].

For the sake of simplicity we shall restrict the discussion which follows to cases when the space describing frustration is of constant curvature.

\section{Decurving curved spaces by disclinations.}

The density of disclinations necessary to decurve a space of sectional curvature $\kappa$ (a constant for all two dimensional sections in a space of constant curvature) is easily obtained through the use of the Gauss-Bonnet theorem applied to a small element of surface $S$ limited by a loop $\gamma$. The quantity $\theta=\int_{\gamma} \kappa \mathrm{d} S$ is an angle which measures the rotation of any vector transported parallel to itself along $\gamma$ in the curved space (for parallel transport, see [8]). $\theta$ is also the order of magnitude of the angle by which an isometric mapping (Ref. [8], p. 78) of $\gamma$ on flat space opens the surface bound by $\gamma(\kappa>0)$ along a cut performed in $S$, if $S$ is infinitesimal. Therefore $\theta$ is a measure of the strength of the wedge disclination which has to be introduced in order to close the gap (see Fig. 1). Clearly, a triple set of wedge disclination lines is necessary to decurve a $3 \mathrm{~d}$ space.

The problem at hand (how to go from curved space to the amorphous system) is better under- 


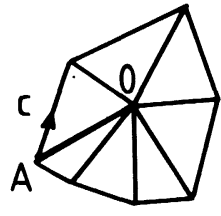

a*)

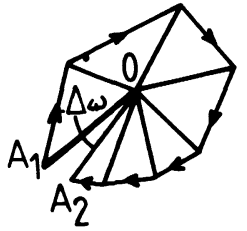

b)

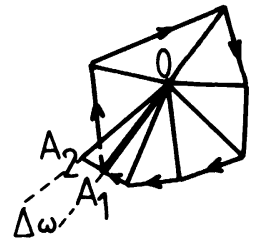

c)

Fig. 1. - Isometric mapping of a loop $\gamma$ on flat space $: a$ ) tri-contour $\gamma$ and surface inside are approximated by a triangulation ; $b$ ) case of a space of positive curvature ; $c$ ) case of a space of negative curvature.

stood if we approximate (triangulate) the curved space by the very polyhedra which describe the local order, for example tetrahedra $\{3,3\}$ in the case of metallic atoms $\left({ }^{1}\right)$. Triangulation of curved spaces has been used by Regge [9] to deal with relativity " without coordinates ».

Note that the sign, and possibly the curvature of the curved space itself, once the building block is defined, can be left at will. According to the choice which is made, this will result in different systems of disclinations to decurve it; for example, even if the local order of metallic atoms is better represented by a spherical packing of 5 tetrahedra around an edge (noted $\{5,3,3\}$, which means that, in terms of Voronoï cells, we have 3 dodecahedra $\{5,3\}$ around each edge of a cell), it might be preferable to embed the tetrahedra in hyperbolic space which has the advantage of being infinite, contrary to the spherical space. Let us choose for the sake of illustration the $\{5,3,4\}$ tessellation, which is hyperbolic with 4 dodecahedra around each edge (we use the Voronoi language). Disclinations introduced along the edges build locally a $\{5,3,3\}$ structure, which is what is expected. However the density of disclinations we have to introduce is very large, since $\{5,3,4\}$ is far from flatness. This fact can be appreciated by noticing that the dihedral angle of a dodecahedron $\{5,3\}$ is $\alpha=116,6 \mathrm{~d}^{\circ}$, which, if one arranges $4\{5,3\}$ around a common edge, yields a surplus angle $\theta_{\mathrm{h}}=360-4 \times 116.6 \sim-106.4 \mathrm{~d}^{\mathrm{o}}$, which is large and of the same order as the dihedral angle $\alpha$. Conversely, the local $\{5,3,3\}$ structure shows a deficit angle $\theta_{\mathrm{s}}=360-3 \alpha \sim+10.2 \mathrm{~d}^{\mathrm{o}}$, which can be considered as small, so that the distortions produced by the disclinations can now be sustained by the material, in real space.

\section{The duality between spherical and hyperbolic local arrangements.}

This example showed that the disordered medium contains regions of spherical "curvature " (along the disclination lines, with local arrangement $\{533\}$ ) and regions of hyperbolic « curvature " (elsewhere, the local arrangement is $\{534\}$ with elastic distortions). The hyperbolic regions show up a surplus angle $\theta_{\mathrm{h}}<0$, while the spherical regions show up a deficit angle $\theta_{\mathrm{s}}>0$. They are therefore the sources of distortions (and internal stresses) of opposite signs. Except if there exist lattice disclinations in the hyperbolic space which yield $\theta_{\mathrm{s}}=0$, it is not possible to avoid these internal stresses. But if per chance $\theta_{\mathrm{s}}=0$, this means that disclinating the hyperbolic lattice decurves it to a flat space without distortions (stresses), in which case the local order is not frustrated. We do not consider such situations, which exist for particular types of tessellations (ex. : $\{435\}$ is hyperbolic and can be decurved to $\{434\}$ which is simple cubic). We are therefore led to the conclusion that an amorphous body possesses, by virtue of our construction which is very general, internal stresses of both signs. Is it possible to minimize these internal stresses by a convenient spatial arrangement of spherical and hyperbolic regions ? The answer is practically given in [1], where we have shown that the disclination lines (of spherical curvature) arrange in a

$\left.{ }^{1}\right)$ We use Schläfli symbols; $\{p, q\}$ is a regular polyhedron with $p$ edges on each face, $q$ faces per vertex. 
connected network, dual to another network of « reticular axes » of a hyperbolic nature. But we can now give a deeper interpretation.

Assuming that the curved space of reference (hyperbolic, say) represents well the local order of the amorphous system (with correct lengths and angles), we noticed in [1] that it is advisable to map isometrically the curved space along sets of closed polygons whose edges are reticular axes of the curved crystal ; the central region of the polygon contains a disclination, since the mapping of the polygon is an open polygon; the neighborhood of the polygon is slightly distorted. The point we want to emphasize is that the distortions (and stresses) which attend the isometric mapping along the polygon are characteristic of an elastic disclination, of angle $\theta_{\mathrm{h}}$ (the surplus angle related to the hyperbolic curvature). By hypothesis, $\theta_{h}$ is small, since the curved space of reference represents well the local order. Similarly, the distortions (and stresses) due to the topological disclination located at the centre of the polygon are characteristic of an elastic disclination of (small) angle $\theta_{\mathrm{s}}$; the strength of the topological disclination is chosen in such a way that $\theta_{\mathrm{s}}$ and $\theta_{\mathrm{h}}$ take opposite signs.

We see therefore that the polygonal lines (which we shall call $C_{\mathrm{h}}$-lines, because the mapping achieved in their vicinity is a kind of cartography of this vicinity), and the disclination lines ( $D_{\mathrm{s}}$-lines) are absolutely dual. The $C_{\mathrm{h}}$-lines could also be considered as the $D_{\mathrm{h}}$-lines built in a spherical space, the $D_{\mathrm{s}}$-lines being the $C_{\mathrm{s}}$-lines of this space. Of course, the construction of the disordered medium starting from a spherical space raises new problems, in particular concerning the fitting of various sphere mappings; but we see that in principle there should be a method yielding the same result as when starting from a hyperbolic space.

\section{Typical size of a polyhedral domain. Influence of $T$. Existence of $T_{0}$.}

A typical size $\xi$ of a cell of the irregular lattice can be easily estimated, using for simplicity a $2 d$ model of parallel disclinations. The elastic energy per unit length of two parallel wedge disclinations of opposite signs and at a distance $\xi$ is, according to reference [10],

$$
W_{\mathrm{ei}}=\frac{\mu \theta^{2} \xi^{2}}{16 \pi(1-v)}\left[1-\frac{4 a_{0}^{2}}{\xi^{2}}\left(\ln \frac{\xi}{a_{0}}\right)^{2}\right],
$$

where $\theta$ is the rotation angle and $a_{0}$ a core radius. By taking for $\theta$ the mean value $\left(\theta_{\mathrm{s}}-\theta_{\mathrm{h}}\right) / 2$, we obtain a reasonable approximation of the elastic energy involved in our model $\left({ }^{2}\right)$. This amounts, per unit volume, to

$$
f_{\mathrm{el}} \simeq \frac{\mu \theta^{2}}{16 \pi(1-v)}\left[1-\frac{4 a_{0}^{2}}{\xi^{2}}\left(\ln \frac{\xi}{a_{0}}\right)^{2}\right] .
$$

To which must be added an energy of line, which takes into account the fact that the coordination numbers are different on the $C$ and the $D$ lines, and a core energy. Let us estimate it to be of the order of $\mu a_{0}^{2} / 10$ per unit length of line [10]. The total energy reads :

$$
f=f_{\mathrm{el}}+f_{\mathrm{c}}=\frac{\mu \theta^{2}}{16 \pi(1-v)}\left[1-\frac{4 a_{0}^{2}}{\xi^{2}}\left(\ln \frac{\xi}{a_{0}}\right)^{2}\right]+\frac{\mu}{10} \frac{a_{0}^{2}}{\xi^{2}},
$$

and is minimized for

$$
\frac{\theta^{2}}{2 \pi(1-v)}\left[\left(\ln \frac{\xi}{a_{0}}\right)^{2}-\ln \frac{\xi}{a_{0}}\right]=\frac{1}{5}
$$

$\left({ }^{2}\right)$ This value of $\theta$, obtained as the mean value of $\left|\theta_{\mathrm{s}}\right|$ and $\left|\theta_{\mathrm{h}}\right|$, is highly debatable. As we shall mention at the end of this paper, the spherical and hyperbolic regions do not occupy equal volumes in the body. Also, averages other than arithmetic averages can be thought of (see Ref. [14]). 
i.e.

$$
\ln \frac{\xi}{a_{0}}=\frac{1}{2}+\frac{1}{2} \sqrt{1+\frac{8 \pi}{5} \frac{1-v}{\theta^{2}}} \sim \frac{+1}{\theta} \sqrt{\frac{2 \pi}{5}(1-v)}+\frac{1}{2}+\frac{\theta}{8} \sqrt{\frac{5}{2 \pi(1-v)}},
$$

for $\theta$ small enough. With $\theta \sim 1 / 5 \mathrm{rad}$, we get $\xi / a_{0} \sim \mathrm{e}^{5} \sim 150$.

We note that the solution of (2) which is here chosen makes $\ln \frac{\xi}{a_{0}}$ positive, and that the equilibrium is (meta)stable.

This very crude calculation, which gives a reasonable characteristic size for the networks, seems also to indicate that this size is very little dependent on the physical elastic constants, but depends rather on the symmetry properties of the short-range order, through $\theta$. The mean stress $\sigma \sim$ $\mu \theta / 2 \pi(1-v)$ is of the order of the yield stress, but this is probably overestimated, since we have taken a model of parallel disclination, while the real system is $\mathbf{3}$ dimensional, which allows for a better screening of the stresses. A more realistic model would also probably yield a larger characteristic length. The total energy is $f \sim \mu / 500$, i.e. $f\left(\xi / a_{0}\right)^{2} a_{0}^{3} \sim \mu a_{0} / 5$ per atom along a disclination. This rather large value (compared to $k_{\mathrm{B}} T_{\mathrm{m}}$ ) indicates that the system we are describing is very far from equilibrium, and that such a metastability can be insured only if the system is frozen at low temperature. It is therefore a model valid below $T_{\mathrm{g}}$.

However, above $T_{\mathrm{g}}$, in the supercooled state, the lines are probably mobile, and the model must be completed by introducing an entropy of configuration of the lines, which yields a free energy term

$$
f_{\text {ent }}=\frac{k_{\mathrm{B}} T}{a_{0}^{3}}[\rho \ln \rho+(1-\rho) \ln (1-\rho)],
$$

where $\rho$, the density of lines is, in our simplified model, of the order of $a_{0}^{2} / \xi^{2}$. Minimizing $f=$ $f_{\mathrm{ei}}+f_{\mathrm{c}}+f_{\mathrm{ent}}$ we find (with $\rho$ assumed small)

$$
\frac{\mu \theta^{2}}{16 \pi(1-v)}(\ln \rho)^{2}+\left(\frac{\mu \theta^{2}}{8 \pi(1-v)}-\frac{k_{\mathrm{B}} T}{a_{0}^{3}}\right) \ln \rho-\frac{\mu}{10}=0 .
$$

When $T$ is small compared to $T_{0}=\mu a_{0}^{3} \theta^{2} / 8 \pi(1-v) k_{\mathrm{B}}$ we recover the former situation. Using $\mu a_{0}^{3} \sim 100 k_{\mathrm{B}} T_{\mathrm{m}}$, we find $T_{0} \sim 0.25 T_{\mathrm{m}}$. Noting $\ln \rho=x$, and assuming $\rho$ small compared to 1 , we have

$$
\frac{T_{0}}{2} x^{2}+\left(T_{0}-T\right) x-10 T_{m}=0 .
$$

The solution which satisfies $\partial^{2} f / \partial \rho^{2}>0$ (stability) is

$$
x=\ln \rho=\frac{1}{T_{0}}\left[T-T_{0}-\sqrt{\left(T-T_{0}\right)+20 T_{0} T_{m}}\right] .
$$

The density at $T=T_{0}$ is very small and of the order of $\mathrm{e}^{-9}$, i.e. $\xi / a_{0} \sim 90$. It increases monotonically with $T$ and reaches at $T=T_{\mathrm{m}}$ a value of the order of $\mathrm{e}^{-3}$, corresponding to a ratio $\xi / a_{0}$ of the order of 5 . The physical value of $\xi / a_{0}$ is that which is obtained at $T_{\mathbf{g}}$, in between these two values. It is clear that the entropy contribution yields a value of $\xi / a_{0}$ much smaller than the value obtained from (3).

If the first term $\frac{T_{0}}{2} x^{2}$ is negligible compared to $\left(T_{0}-T\right)|x|$ we obtain a Vogel-Fulcher law, 
with $T_{0}$ being interpreted as the critical temperature at which the configurational entropy vanishes. This approximation requires

$$
T_{\mathrm{m}}>T>T_{0}\left(1+\frac{|x|}{2}\right),
$$

i.e., with the above values,

$$
|x|<6
$$

which is clearly incompatible with the solution $|x|=\frac{10 T_{\mathrm{m}}}{T-T_{0}}$, except largely above $T_{\mathrm{m}}$ but anyway indicates a tendency towards a V.F law at high temperature of the supercooled liquid.

Our result which, as expected, departs from the classical Arrhenius law, also shows a variation with temperature ( $\mathrm{d} \ln \rho / \mathrm{d} T>0$ ) which is found in all those kinds of theories [11, 12], the first one of this type having been proposed by Anderson [11] who introduced defects whose nature was not defined, but with a logarithmic energy rather than a logarithm-squared energy as in the present work. Rivier's paper [12], while more in the line of this work (his defects are disclinations of an elastic medium), did not foresee their mutual arrangements, and also attributed to them a logarithmic energy. This paper seems therefore the first attempt to give to $T_{0}$ a structural explanation, which in fact differs from the previous ones, since our $T_{0}$ (because of the logarithm-squared term) does not appear as a critical point. However our oversimplified model clearly needs further development :

a) we have limited our calculation to a $2 \mathrm{~d}$ case; the stresses would be further screened in the real 3d case, and the logarithm squared term could lose importance (see also [12]);

b) dynamical phenomena are to be introduced in the supercooled state, where lines are probably mobile, by mechanisms which are worth studying in detail, in particular the thermally excited emission of dislocation lines ;

c) last but not least (Friedel, private communication), we have not taken into account the (negative) energy contribution which favours the curved state, i.e. the tendency in a metallic glass to have locally more than 12 neighbours (positive curvature) or in a covalent glass to form 7-bond rings, say (negative curvature). This contribution is counterbalanced by the positive elastic energy, and the competition between these two terms defines indeed the frustrated situation. R. Kerner [14] has recently calculated the negative energy term of a specific $2 \mathrm{~d}$ model, and shown how it can favour the amorphous versus the crystalline state, but he does not introduce any elastic contributions.

There are big conceptual difficulties in evaluating this negative "potential of curvature "

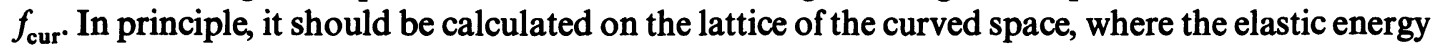
vanishes by definition, but since the minimum of the total energy must be reached in flat space, this is not physical. It is therefore expected that the variable which enters this energy $f_{\text {cur }}$ is some angle $\theta$, analogous to the angle used in $f_{\mathrm{e}}$ (see also [14]). But $f_{\text {cur }}$ would be minimized for a nonvanishing value $\theta_{0}$, of the order of $\theta_{s}$ or $\theta_{h}$.

In any case, this potential of curvature could not be very large, since spherical and hyperbolic regions, which bring opposite contributions to $f_{\text {cur }}$, are present at the same time in an amorphous body. We therefore leave the discussion of $f_{\text {cur }}$ to a further study. We notice anyway an unexpected conclusion of these remarks : the core of a crystal dislocation is often described as an amorphous region; since its size can be much smaller than $\xi$, a single type of curvature can be present in the core, and it is therefore not impossible that in certain circumstances the core energy be negative in a crystal. 


\section{Long-range orientational correlations.}

If one believes in the principles of the " best " packing we have discussed in section 3 (these principles have not to be fulfilled exactly in a real situation), the polygonal contours ( $C$-lines, say) which surround a spherical line ( $D$-line, say) have a symmetry of a polygon liable to appear in a spherical tessellation, and the polygonal contours ( $D$-line) which surround a hyperbolic line $(C$ line) have a symmetry of a polygon liable to appear in a hyperbolic tessellation. For example the $C$-lines surrounding a disclination of pentagonal symmetry form pentagons. This suggests that the conjugated networks of $C$ - and $D$-lines build themselves a disordered system of segments, which is describable in terms of disclinations at a higher scale, and so on... In other words it should not be possible to define a (Euclidean) usual crystal as a $3 \mathrm{~d}$ repeat of a disclinated unit cell, in such a way that the infinite crystal is the image, in a suitable decurving process, of a hyperbolic lattice on $\mathbf{H}^{3}$, or the sum of suitably disclinated lattices on spheres $\mathrm{S}^{3}$.

Another way of getting this result is as follows. Suppose we are able to achieve a (Euclidean) crystal by fitting together equal spheres $S^{3}$ previously decurved by wedge disclinations. Suppose also this fitting leaves no voids or overlappings of matter, does not create singular walls at the interfaces of contact and has only introduced some elastic deformations on the decurved spheres. Therefore these decurved spheres, which form the building blocks of the crystal, are homotopic to tori $\mathrm{T}^{3}$, by identifying faces of these blocks corresponding to periodic boundary conditions. We must therefore conclude that there is a change in topological properties (from a $S^{3}$ to a $T^{3}$ ) introduced by the decurving process. But this would require more drastic operations than the simple introduction of wedge disclinations inside the $S^{3}$ lattice, because wedge disclinations do not change the topology. We have therefore to look for operations of a different nature than those discussed in this paper in order to obtain a Euclidean crystal from a decurving process of a crystal in curved space.

However, there should subsist some kinds of correlations between the orientations in the final amorphous body. Neighboring segments of $C$ - or $D$-lines are correlated (but to an elastic distortion, which insures disorder in the long range). The nature of the correlations (probably decaying algebraically) has still to be worked out. But at this stage our model seems to be in agreement with the computational results of [13] concerning icosahedral correlations.

Our last remark concerns the relative extent of the hyperbolic and spherical regions in the amorphous body; on one of the networks the density is much larger than average, and on the other one much smaller. There are all reasons to believe that the regions with a larger density (hyperbolic) have a lesser extent, so that the two conjugated networks must be quite different spatially. Also, the "cores » of the hyperbolic disclinations can be extended or split in a way which reduces the local density.

\section{Acknowledgments.}

We have benefited from interesting discussions with Prof. J. Friedel.

\section{References}

[1] KLÉman, M., J. Physique 43 (1982) 1389.

[2] Finney, J. L., Proc. Roy. Soc. London A 319 (1970) 479.

[3] Kléman, M. and Sadoc, J. F., J. Physique Lett. 40 (1979) L-569.

[4] Farges, J., De Féraudy, M. F., Raoult, M. F. and Torchet, G., J. Physique Colloq. 36 (1975) C2-13.

[5] Steinhardt, P. and Chaudhari, P., Philos. Mag. A 44 (1981) 1375.

[6] Toulouse, G., Commun. Phys. 2 (1977) 115. 
[7] Bilby, B. A., Prog. Solid Mech. 1 (1960) 329.

[8] CARTAN, E., Leçons sur la géométrie des Espaces de Riemann (Gauthier-Villars, Paris), 1963.

[9] ReGGe, T., Il Nuovo Cimento 19 (1961) 558.

[10] Friedel, J., Dislocations (Pergamon Press) 1964.

[11] ANDERson, P. W., Ill-condensed matter, Les Houches 1978, Session XXXI, R. Balian, R. Maynard and G. Toulouse, eds (North-Holland) 1979.

[12] Rivier, N. and Duffy, D. M., Critical phenomena, J. Della Dora, J. Demongeot and B. Lacolle, eds., Synergetics, Vol. 9 (Springer) 1981.

[13] Steinhardt, P. J., Nelson, D. R. and Ronchetti, M., Phys. Rev. Lett. 47 (1981) 1297.

[14] Kerner, R., Phenomenological Lagrangian for the amorphous solid state, preprint.

Kerner, R. and Dina Dos Santos, C. R. Hebd. Sean. Acad. Sci. 295 (1982) 1055. 